\title{
DISCRETE DYNAMICAL MACROMODELS AND THEIR USAGE IN ELECTRICAL ENGINEERING
}

\author{
Petro Stakhiv, Yuriy Kozak \\ Lviv Polytechnic National University, \\ 12 St. Bandera St., 79013 Lviv, Ukraine, \\ e-mail:spg@polynet.lviv.ua, ykozak@mail.ru
}

\begin{abstract}
An optimization approach intended for construction of macromodels of dynamic objects especially for electrotechnical systems is proposed in the paper. This approach is efficient because of its universality. A set of techniques to simplify optimization task needed for the identification of macromodels is considered. The specifics of the use of the mentioned approach for the creation of models for autonomous objects and prediction of dynamic processes are described. The test example of the macromodel construction for two-winding transformer using the considered approach is presented.
\end{abstract}

Keywords: mathematical models, optimization, parameters identification.

\section{INTRODUCTION}

Macromodel is a simplified mathematical representation of some object or process that is designed to describe only a subset of object characteristics which are required for particular analysis. Generally macromodel describes only external parameters of the object it represents, and does not describe its internal processes. Replacing real objects with their macromodels significantly reduces the complexity of simulation by ignoring processes we are not interested in.

Macromodels of components of electrotechnical and power systems are widely used in modern simulation and design systems. This is caused by complexity of simulated systems and variety of physical phenomena that should be taken into account during simulating or designing of a complex system. Macromodels are very useful in such conditions because of their simplicity, and it concerns especially the dynamical macromodels in the form of discrete state variables:

$$
\left\{\begin{array}{l}
\vec{x}^{(k+1)}=F \vec{x}^{(k)}+G \vec{v}^{(k)}+\vec{\Phi}\left(\vec{x}^{(k)}, \vec{v}^{(k)}\right) \\
\vec{y}^{(k+1)}=C \vec{x}^{(k+1)}+D \vec{v}^{(k+1)}
\end{array}\right.
$$

where $\vec{v}$ - variables which describe external influences; $\vec{y}$ - variables which describe output values; $\vec{x}$ - variables which describe the object state; $F, G, C, D$ - some matrices with unknown coefficients which should be found, $\Phi-$ a vectorfunction, the form and coefficients of which should also be found; $k$ - the discrete number. This form of the macromodel representation can be used for wide variety of objects and is convenient for usage in computer applications.

Macromodels construction is a nontrivial task. There are many approaches to solve it, though most of them apply significant limitations on the set of objects for which a model can be built or are too complex for practical usage. One of the most popular algorithms for linear dynamic macromodel identification in a form of discrete state variables is a Ho-Kalman's algorithm, which was improved for bilinear form of discrete state variables by Isidori [1]. Nevertheless, the mentioned algorithms except the limitations concerning the mathematical form of macromodel representation have two more significant disadvantages:

1. They apply strict requirements on the set of experimental data which are used for macromodel identification.

2. They are very sensitive to the noise, which may be present in experimental data. The matrix factorization task becomes an ill-conditioned problem in this case.

One of the most promising approaches for construction of macromodels for a wide range of objects is a usage of optimization. This approach is stable and correct, and does not apply any limitation on modeled object, which makes it useful for 
identification of macromodels in any form in case it is defined by finite number of real coefficients. One more advantage of this approach is a possibility to use any transient characteristics, which is important for identification of macromodels of complex objects. The main its disadvantage is a complexity of optimization task to which macromodel identification is reduced.

This paper presents an analysis of efficiency of optimization approach for identification of macromodels, denotes problems which might happen in its practical usage, presents a practical recommendations for effective usage of proposed approach for construction of macromodels of components of electrotechnical systems.

\section{THE IDEA OF MACROMODEL IDENTIFICATION USING OPTIMIZATION}

Let's consider some dynamical object, which is schematically shown in the fig. 1, for which a macromodel in a form (1) is built.

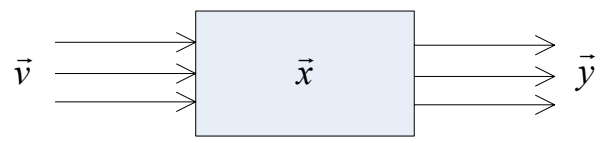

Fig. 1 - An object for the macromodel construction

Let's assume, that we know some binary relation between discrete sets of input variables, which cause some transient processes in the object $\left\{\vec{v}_{i}^{(k)}\right\}$ and corresponding object responses $\left\{\vec{y}_{i}^{(k)}\right\}$, where $k-\mathrm{a}$ number of discrete, $i-$ a number of process. Let's define a goal function, which expresses the inaccuracy of object behavior simulation with the use of obtained macromodel. In a simplest case it can be a root-mean square deviation:

$$
Q(\vec{\lambda})=\sum_{i} \sum_{k}\left(\overrightarrow{\tilde{y}}_{i}^{(k)}-\vec{y}_{i}^{(k)}\right)^{2}
$$

where $\overrightarrow{\tilde{y}}_{i}^{(k)}$ - object's response, calculated using macromodel, $\vec{y}_{i}^{(k)}$ - real object response, $\vec{\lambda}$ - a set of macromodel parameters. In case macromodel is built in a form (1) vector $\vec{\lambda}$ will consist of elements of matrixes $F, G, C, D$ and coefficients, which describe non-linear function $\Phi(\cdot)$. The set of coefficients $\vec{\lambda}^{*}$ where function (2) reaches its minimum will be an optimal set of macromodel coefficients. Thus macromodel identification can be reduced to finding of the function (2) minimum point.
It should be pointed out, that elements of vector $\vec{x}$ will have unknown physical sense during such macromodel identification. In some cases elements of vector $\vec{x}$ can be combinations of values with different measurement units.

In spite of advantages of the considered approach, mainly its universality, it has a serious disadvantage - complexity of optimization task. Numerical determination of minimum of nonlinear function of many variables, which is defined in a numerical form, is a complex mathematical problem [2]-[5]. We will skip all details here, and only consider those characteristics of our optimization task, which allows us to select the most suitable algorithm for our optimization task.

Optimized function in our case often contains socalled narrow ravines - dependencies written down in the following form:

$$
Q(x, y)=C_{x} \cdot x^{2}+C_{y} \cdot y^{2} ; C_{x}<<C_{y}
$$

First of all such form of the goal function is caused by big difference in level of dependency of model behavior from different coefficients. This dependency is often different in different object states that makes mentioned narrow ravines to become bent and leads to increasing of the optimization task complexity.

For most optimization algorithms such goal function causes significant decrease of the algorithm step that leads to considerable increasing of computation time or even to the algorithm failure. That it why on practice it is recommended to use algorithms which works well for goal functions with clearly expressed ravines. One more aspect which should be taken into account when selecting the optimization algorithm is computation errors. In optimization task computation errors cause a large number of small local minimums, which may result stoppage of optimization algorithm.

The most perspective algorithms from this point of view are stochastic algorithms. Authors used Rastrigin's directing cone method [3] with step length and cone angle adaptation [5], dynamic scaling of parameters space and procedure for exiting from local minimums.

The usage of specialized optimization algorithm itself does not solve the problem with complexity of optimization task. There is a variety of other approaches intended for this problem solving and which can be combined:

- simplification of optimization task by its dimension decreasing:

0 by splitting of the macromodel identification procedure into stages [6]; 
o by determining of some macromodel coefficients using expert analysis [6];

0 by introducing of additional input variables in order to simplify nonlinearity with the subsequent analytical derivation of resulting macromodel [7];

- using parallelization of calculations in order to use computation power of many CPU cores [8];

- gradual increase of macromodel complexity [9].

Mentioned approaches help to solve the optimization task, though they require creativity and are difficult for automation, though many of them theoretically can be fully or partially automated. Automation of different approaches of simplification of optimization task to be solved for macromodel construction requires future development.

\section{ADEQUACY OF THE OBTAINED MACROMODEL}

During construction a macromodel of nonlinear object we always should consider the adequacy of obtained model. This question should be answered for nonlinear models only as we have no guarantee, that model will be able to simulate correctly the required process, which was not included into the set of processes used for the macromodel construction. There are several ways to answer this question. Most common way is to test the obtained macromodel on independent process or set of processes. Such verification increases the confidence that the model is adequate, though can't guarantee its adequacy.

An alternative approach to answer this question is to use expert analysis for evaluation of the nonlinearity nature. Using appropriate mathematical form for estimation of nonlinear function $\Phi$ we can obtain a macromodel with high probability of its adequacy.

It is obvious that mentioned approaches can be combined, that allows to increase the confidence that obtained model is adequate.

\section{INITIAL STATE AND AUTONOMOUS MACROMODELS}

Some more problems arise in case if modeled object can be in different initial states [9]. Such situation happens if modeled object includes mechanical elements, which can be in different positions, could have residual magnetization etc. In particular, it can happen is case of autonomous objects, for which different initial state can be the only reason of their different behavior. For autonomous models equation (1) can be rewritten in the following form:

$$
\left\{\begin{array}{l}
\vec{x}^{(k+1)}=F \vec{x}^{(k)}+\vec{\Phi}\left(\vec{x}^{(k)}\right) \\
\vec{y}^{(k+1)}=C \vec{x}^{(k+1)}
\end{array}\right.
$$

For discrete form of state variables (1), (4) the initial state of modeled object is depicted by a value of zero discrete of state vector $\vec{x}$. So components of this vector should be included into the set of unknown macromodel coefficients $\vec{\lambda}$. The value of zero discrete of state vector $\vec{x}$ can be different for different processes. To account this we might build vector of unknown coefficients $\vec{\lambda}$ from two parts: $\vec{\lambda}_{\text {fixed }}$, which includes those model coefficients, which are the same for all processes, and $\vec{\lambda}_{\text {free }}$, which includes an independent copy of zero discrete of vector $\vec{x}$ for each process. This change significantly increases the number of coefficients, which should be found by optimization, and thus increases the complexity of optimization task.

For practical usage of the obtained macromodel we need to have a way to determine zero discrete of vector $\vec{x}$ from some data. For example, it can be several discretes of output variables. An additional dependency should be identified in this case:

$$
\vec{x}=\vec{f}\left(\vec{y}^{(1)}, \vec{y}^{(2)}, \ldots, \vec{y}^{(l)}\right)
$$

where $l-\mathrm{a}$ number of output discretes used to determine zero discrete of vector $\vec{x}$.

Optimization approach, due to its universality, is perfect one for identification of such additional dependencies. In fact, it means that elements of vector $\vec{x}$, which were added to the set of unknown coefficients $\vec{\lambda}$ should be replaced with coefficients of function $\vec{f}(\cdot)$.

\section{INPUT INFORMATION AND ITS INFLUENCE ON THE PROCESS OF MACROMODEL CONSTRUCTION}

There are two factors, which can influence the choice of processes, which will be used as input information for macromodel construction:

1. Input information should describe the modeled object well enough. We are considering a nonlinear object, so exact simulation of one set of processes does not guarantee precision for another one. Thus input information should be wide, and, theoretically, should include all possible processes. So it should include unlimited number of processes, which is impossible. A great number of processes also slow down the optimization algorithm because it complicates the 
calculation of the goal function.

2. Insufficient number of processes leads to inadequacy of obtained macromodel.

Mentioned two factors apply contradicting requirements on the input information. Thus a selection of input information is a compromise between the time needed for the macromodel construction and quality of the result. We should note here, that the macromodel itself is a compromise between precision and complexity of the model.

The following rules are used on practice to select input information:

- All significantly different operating modes should be included;

- We are considering a dynamical object, so it is advisable to use rapid changes of input variables, because they better expose the transient characteristics of the object.

- It is not recommended to include similar processes.

\section{USAGE OF MACROMODELS FOR DYNAMICAL PROCESSES FORECAST}

Dynamical processed forecast, as a mathematical problem, often appears in different areas of human life. It is most typical for economical processes, where forecasting is one of the most often tasks. There are two different cases:

1. Time interval, for which a forecast should be done, is a continuation of the interval where object's behavior is known. This is the most typical situation for economics. Here we need to do a forecast values for the future in case we know values for the past. In this case zero discrete of vector $\vec{x}$ can be found by including it into vector $\vec{\lambda}$.

2. Time interval, for which a forecast should be done is independent, and is not a continuation of the interval where object's behavior is known. Such situation is common for periodical processes. For example when we know permonth values of some economical characteristics for several years, and we need to forecast it for some another year [9]. In this case we need to determine some additional dependency, which will allow us to determine zero discrete of vector $\vec{x}$. So we need to find the function (5), mentioned above.

\section{AN EXAMPLE OF MACROMODEL CONSTRUCTION}

Authors constructed a number of macromodels of different dynamic objects, such as transformers, electromechanical converters and electric motors in general, voltage regulators, some economical processes [9]-[12]. For example, now we are presenting a macromodel of two-winding transformer for output stage of audio frequency amplifier with a permalloy core.

As input variables for this model were selected voltages on both windings, corresponding currents were selected as output variables. The model was based on experimentally measured transient characteristics - currents in both windings were measured when constant voltage was applied to one winding and shorted another winding. Thus two experiments were done: one for constant voltage applied to primary winding and shorted secondary winding (see Fig. 2-3), and another one for constant voltage applied to secondary winding and shorted primary winding (see Fig. 4-5). Measurement frequency was $8 \mathrm{kHz}$.

Coefficients identification was done with the usage of modified Rastrigin's directing cone method, mentioned above. The form of nonlinearity was selected in accordance to its physical nature in the following form:

$$
\vec{\Phi}\left(\vec{x}^{(k)}, \vec{v}^{(k)}\right)=\left(\begin{array}{c}
\alpha_{1}\left(x_{1}^{(k)}\right)^{2} v_{1}^{(k)}+\alpha_{2}\left(x_{1}^{(k)}\right)^{3} \\
\alpha_{3}\left(x_{2}^{(k)}\right)^{2} v_{2}^{(k)}+\alpha_{4}\left(x_{2}^{(k)}\right)^{3}
\end{array}\right)
$$

Where $\alpha_{1}, \alpha_{2}, \alpha_{3}, \alpha_{4}-$ unknown coefficients which should be found; $\vec{x}^{(k)}=\left(\vec{x}_{1}^{(k)}, \vec{x}_{2}^{(k)}\right)^{T}$, $\vec{v}^{(k)}=\left(\vec{v}_{1}^{(k)}, \vec{v}_{2}^{(k)}\right)^{T}, \vec{v}_{1}^{(k)}$ and $\vec{v}_{2}^{(k)}$ are voltages on primary and secondary windings correspondingly.

To simplify optimization task several assumptions about coefficient values were applied: all elements of matrix $G$ and non-diagonal elements of matrix $F$ were set to constant values and were excluded from the optimization process.

Obtained macromodel has the following form:

$$
\left\{\begin{aligned}
\vec{x}^{(k+1)} & =\left(\begin{array}{cc}
0.992 & 0 \\
0 & 0.996
\end{array}\right) \cdot \vec{x}^{(k)}+\left(\begin{array}{cc}
0.05 & 0 \\
0 & 0.05
\end{array}\right) \cdot \vec{v}^{(k)}+ \\
& +\left(\begin{array}{c}
0.0048 \cdot \vec{v}_{1}^{(k)}-0.00048 \cdot \vec{x}_{1}^{(k)} \\
0
\end{array}\right) \cdot\left(\vec{x}_{1}^{(k)}\right)^{2} \\
\vec{y}^{(k+1)} & =\left(\begin{array}{cc}
1 & 28.3 \\
15.3 & -0.13
\end{array}\right) \cdot \frac{\vec{x}^{(k+1)}}{1000}+\left(\begin{array}{cc}
9.47 & -33.2 \\
-15.1 & 19.3
\end{array}\right) \cdot \frac{\vec{v}^{(k)}}{100}
\end{aligned}\right.
$$


Where $\quad \vec{y}^{(k)}=\left(\vec{y}_{1}^{(k)}, \vec{y}_{2}^{(k)}\right)^{T}, \quad \vec{y}_{1}^{(k)} \quad$ and $\quad \vec{y}_{2}^{(k)} \quad$ are currents in primary and secondary windings correspondingly.

Output variables, measured experimentally and calculated using obtained model for transient characteristics used for model coefficients identification are shown in the Fig. 2-5.

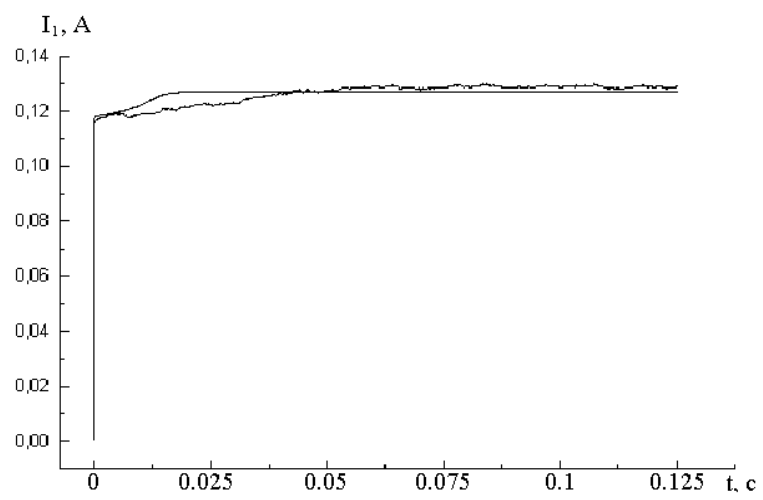

Fig. 2 - Current in primary winding when constant voltage is applied to primary winding and secondary winding is short-circuited

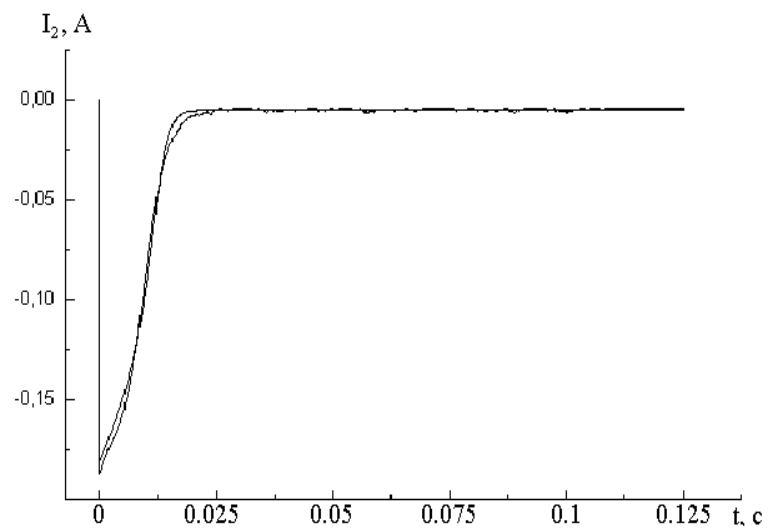

Fig. 3 - Current in secondary winding when constant voltage is applied to primary winding and secondary winding is short-circuited

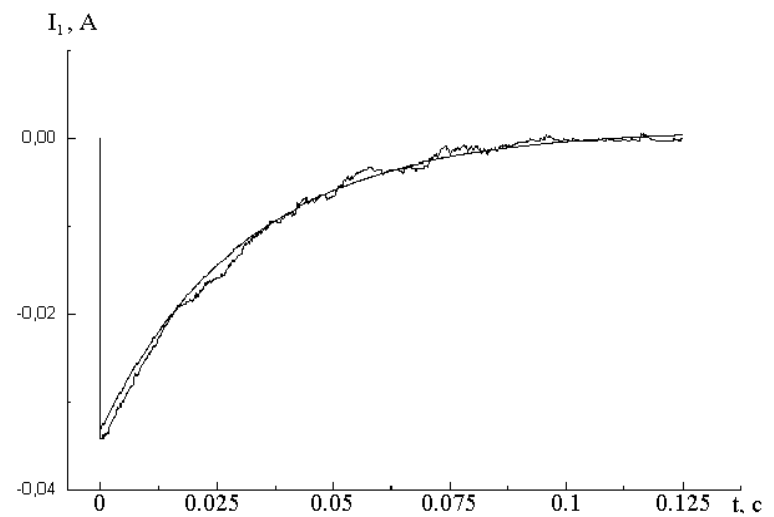

Fig. 4 - Current in primary winding when constant voltage is applied to secondary winding and primary winding is short-circuited

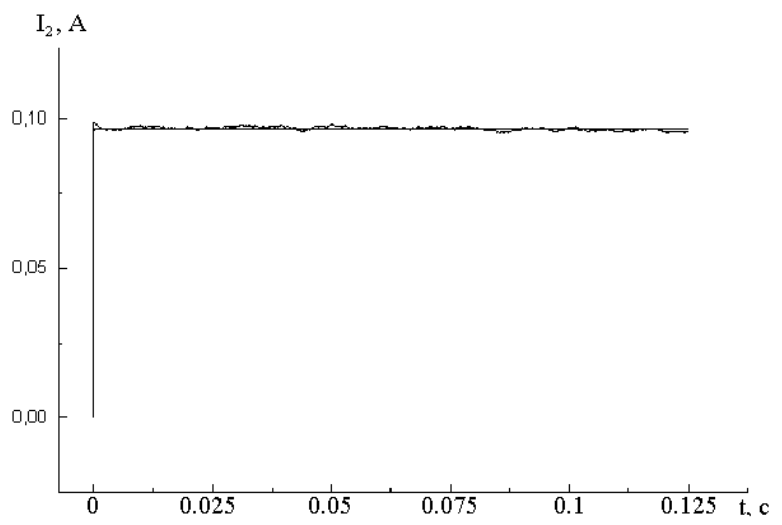

Fig. 5 - Current in secondary winding when constant voltage is applied to secondary winding and primary winding is short-circuited

One more experiment was done for model verification. A voltage on secondary winding of the transformer when sinusoidal voltage with amplitude of $2.6 \mathrm{~V}$ and $17.4 \mathrm{~Hz}$ frequency was applied to its primary winding was calculated using obtained model and compared with experimental data. The result is shown in the Fig. 6.

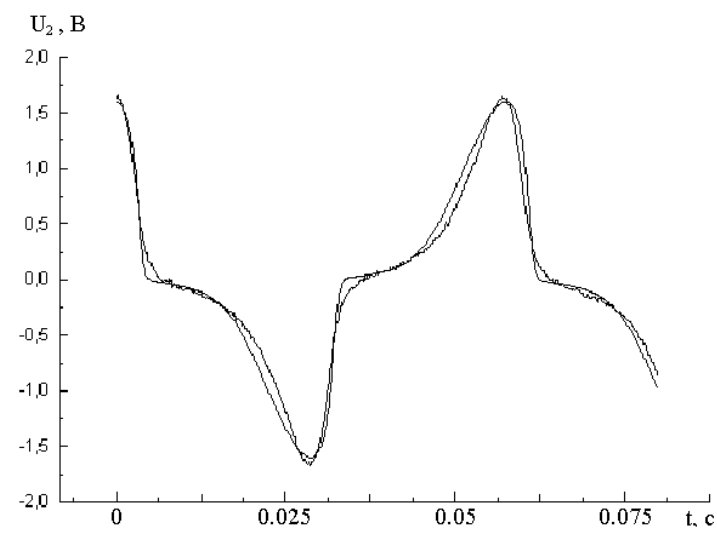

Fig. 6 - Voltage on secondary winding when 2.6V 17.4 Hz sinusoidal signal is applied to primary winding

Good precision of simulation of completely different process allows asserting, that obtained macromodel is an adequate one.

\section{CONCLUSION}

Optimization approach used to construct macromodels for nonlinear dynamical objects is very promising because of its universality. The main disadvantage of this approach is a complexity of optimization task. The set of approaches suitable for solving of this problem are known, but they require human's participation and are complicated for automation. All this allows to state that optimization approach is efficient for construction of macromodels of complex nonlinear dynamical objects, though it needs further development in order to automate macromodel construction process. 


\section{REFERENCES}

[1] Isidori A., Direct construction of minimal bilinear realization from nonlinear input-output maps, IEEE, AC-18 (6) (1973). - pp. 626-631.

[2] Gill P.E., Murray W., Wright M.H., Practical optimization, London, New York: Academic Press, 1986.

[3] Rastrigin L. A., Adaptation of Complex Systems, Riga: Zinatne, 1988 (in Russian)

[4] Shaw A. K., Optimal identification of discretetime systems from impulse response data, IEEE Trans. Signal Process, 42 (1994). - pp. 113120.

[5] Kozak Y. Y. Modification of Rastrigin's directing cone method, Electronics and Communications, Series "Problems of Physical and Biomedical Electronics", (1997). - pp. 424 (in Ukrainian)

[6] Yu. Kozak, B. Melnyk, P. Stakhiv, Simplification of optimization process during mathematical models creation, Przeglad Elektrotechniczny, (12) (2008). - pp. 281-283.

[7] Yu. Kozak, M. Seheda, P. Stakhiv, Mathematical macromodel of turbogenerator based on experimental data, VI Konferencja NaukowoTechniczna SIECI'2008, Szklarska Poręba, Polska, (10-12 września 2008). pp. 411-414.

[8] Liliana Bychkovska-Lipinska, Petro Stakhiv, Yuriy Kozak, Parallelization of calculations in construction of mathematical models using optimization, Proceedings of XIII International Conference "System Modeling and Controll" SMC'2009, ISBN 978-83-927875-0-1

[9] Y. Kozak, B. Melnyk, Yo. Selepyna, Accelerating of the process of discrete macromodels identification by gradual increase in their complexity, Proc. of International Workshop CPEE'2010 "Computational Problems of Electrical Engineering”, Lazne Kynzvart, (13-16 September 2010). - p. 64.

[10] P. Stakhiv, Yu. Kozak, Discrete models of autonomous dynamical systems, Polish Journal of Environmental Studies, 17 (2A) (2008). pp. 77-80.

[11] Kozak Y., Pushchalo Y., Stakhiv P. Construction of discrete macromodel of nonlinear transformer based on experimentally measured transient characteristics, Electronics and Communications, (5) (1998). - pp. 15-18. (in Ukrainian)

[12] P. Stakhiv, Yu. Kozak, Design of discrete models of complex dynamic systems based on optimization approach, Proceedings of the 11th International Conference on "System modeling
Control", Zakopane, Poland, (17-19 October 2005). - pp. 297-301.

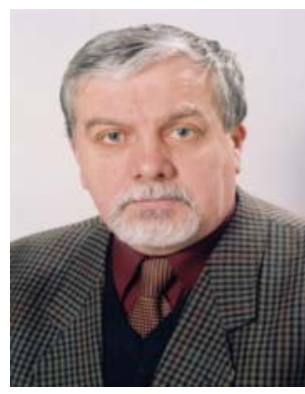

Petro Stakhiv, Ph.D., D. Sc., Professor born in 1948 in Lviv region, Ukraine. He graduated from Physical Faculty of Lviv State University in 1970 and received the M.Sc. degree in radiophysics and electronics. Since 1970 till 1973 he was a Ph.D. student at Department of Theoretical Fundamentals of Electro and Radiotechnics. In 1975 he received the Ph.D. degree in theoretical electrical engineering. The theme of his Ph.D. work was "Synthesis of linear electric circuits (method of state variables)". In 1992 he received D.Sc. degree in the same specialty after doctor thesis defend on "Analysis of dynamic regimes in electric and electronic circuits with multipolar elements". Since 1973 till 1996 he was assistant, associate professor, professor and Head of Department of Theoretical Fundamentals of Electro and Radiotechnics at Lviv State University. In 1996 he began working at Lviv Polytechnic National University as professor and Head of Department of Theoretical and Electrical Engineering.

His scientific interests are mainly concerned with mathematical modeling and simulation of dynamic processes in electrotechnical systems, numerical methods, methods of optimization, system theory and parallel programming.

He proposed different numerical procedures for calculation of dynamic processes on the basis of diacoptic approach. He developed an original method for discrete mathematical model synthesis of multipolar systems using optimization.

$\mathrm{He}$ is the author and co-author of more than 220 publications including 4 monographs, articles and conference proceedings.

Under his supervising 13 Ph.D. and 2 D.Sc. dissertations were prepared. He is an IEEE member and member of Editorial Boards of 2 scientific journals.

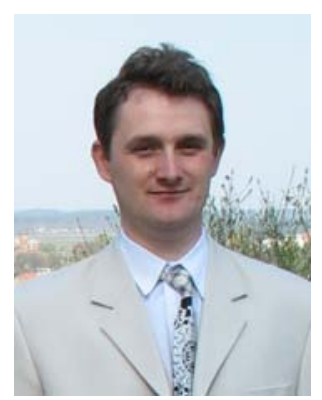

Yuriy Kozak, Ph.D. born in 1975 in Lviv region, Ukraine. He graduated from Physical Faculty of Lviv State University in 1997 and received the M.Sc. degree in radiophysics and electronics. Since 1997 till 2000 he was a Ph.D. student at Department of Theoretical Fundamentals of Electro and Radiotechnics. In 2002 he received the Ph.D. degree in theoretical electrical engineering. The theme of his Ph.D. work was "Construction of mathematical models of components of electrotechnical systems". 
Since 2004 till 2010 he was an associate professor at Department of Theoretical and Electrical Engineering at Lviv Polytechnic National University. Since 2010 he is a D.Sc. student at the same department.

His scientific interests are concerned with mathematical modeling and simulation of dynamic processes in electrotechnical systems, numerical methods, methods of optimization and parallel programming.

He is the author and co-author of more than 25 publications including articles and conference proceedings. 